\title{
Lacticaseibacillus rhamnosus GG inhibits infection of human keratinocytes by Staphylococcus aureus through mechanisms involving cell surface molecules and $\mathrm{pH}$ reduction
}

\author{
I. Spacova ${ }^{1}, C^{\prime} \mathrm{O}^{\prime} \mathrm{Neill}^{2}$ and S. Lebeer ${ }^{1 *}$ \\ ${ }^{1}$ Laboratory of Applied Microbiology and Biotechnology, ENdEMIC group, University of Antwerp, Groenenborgerlaan \\ 171 G.V.522, Antwerp, 2020, Belgium; ${ }^{2}$ Division of Musculoskeletal E Dermatological Sciences, University of Manchester, \\ Oxford Road, Manchester, M13 9PT, United Kingdom; sarah.lebeer@uantwerpen.be
}

Received: 17 April 2020 / Accepted: 2 June 2020

(c) 2020 Wageningen Academic Publishers

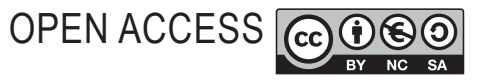

RESEARCH ARTICLE

\begin{abstract}
Beneficial bacteria represent an emerging tool against topical diseases, including infection caused by Staphylococcus aureus. Here, we investigated several anti-pathogenic mechanisms of the model probiotic Lacticaseibacillus rhamnosus GG against a clinical S. aureus isolate by implementing various mutants lacking important cell surface molecules. We analysed adhesion of L. rhamnosus and competitive adhesion with $S$. aureus to primary human keratinocytes, L. rhamnosus and S. aureus auto- and co-aggregation, S. aureus growth inhibition, keratinocyte viability increase, and monocyte Toll-like receptor (TLR) activation by L. rhamnosus as such, or with S. aureus. $L$. rhamnosus mutated in SpaCBA pili exhibited reduced adhesion to keratinocytes, reduced ability to prevent S. aureus adhesion to keratinocytes and reduced co-aggregation with $S$. aureus. Mutants in cell wall exopolysaccharides showed enhanced adhesion to keratinocytes and TLR activation in monocytes, suggesting involvement of additional cell surface molecules masked by exopolysaccharides. All L. rhamnosus strains inhibited S. aureus growth, likely due to acidification of the medium. Live (but not UV-inactivated) L. rhamnosus significantly reduced inflammatory TLR activation in monocytes by $S$. aureus. These data suggest the key role of SpaCBA pili and additional contribution of other cell surface molecules as well as secreted components of L. rhamnosus GG in the multifactorial inhibition of $S$. aureus adhesion and toxicity in the skin niche.
\end{abstract}

Keywords: probiotic, LGG, skin, antipathogenic, pili

\section{Introduction}

Topical application of live probiotic bacteria or derivatives thereof is increasingly explored as a preventive and therapeutic option against skin diseases. Several studies have demonstrated the in vitro effectiveness of probiotic lactobacilli and their products against skin pathogens, such as Pseudomonas aeruginosa (Ramos et al., 2015), Staphylococcus aureus (Mohammedsaeed et al., 2014) and Cutibacterium acnes (Lebeer et al., 2018b). Furthermore, beneficial effects in clinical trials including patients with skin burns (Peral et al., 2009) and acne (Lebeer et al., 2018b) have been reported.
The model probiotic strain Lacticaseibacillus rhamnosus GG represents a promising live skin probiotic for application in humans (Lebeer et al., 2018b). L. rhamnosus GG is widely studied and used in various body niches, where it demonstrates anti-pathogenic and immunomodulatory properties, as well as a favourable safety profile, and robust viability and adhesion to host cells (reviewed by Segers and Lebeer, 2014). Anti-pathogenic mechanisms of $L$. rhamnosus GG include prevention of pathogen biofilm formation (Petrova et al., 2016; Spacova et al., 2018) competitive adhesion to host cells (Mohammedsaeed et al., 2014; Van den Broeck et al., 2018), inhibition of bacterial pathogen growth (De Keersmaecker et al., 2006; 
Van den Broeck et al., 2018) and reduction of pathogenic structure morphogenesis, such as hyphae formation of the fungal pathogen Candida albicans (Allonsius et al., 2019). L. rhamnosus GG was specifically shown to reduce adhesion of the prominent pathogen S. aureus to human intestinal mucus (Vesterlund et al., 2006) and primary skin cells (Mohammedsaeed et al., 2014), however the contributing probiotic molecules have not yet been elucidated.

L. rhamnosus GG mechanisms of action have mostly been studied in the gastrointestinal niche, and several molecular structures of L. rhamnosus GG were previously suggested to promote its probiotic interactions. The SpaCBA pili consisting of three pilin subunits (SpaA, $\mathrm{SpaB}$ and $\mathrm{SpaC}$ ) are especially relevant for probiotic-host interactions, and represent one of the first examples of pili in probiotic lactobacilli (Kankainen et al., 2009; Lebeer et al., 2012). Their potential role in the gastrointestinal tract has previously been described in relation to biofilm formation and efficient adhesion to human intestinal epithelial cells (Lebeer et al., 2012; Spacova et al., 2018) and murine macrophages (Vargas-Garcia et al., 2015), as well as in immunomodulation of intestinal epithelium (Lebeer et al., 2012). Other molecules of L. rhamnosus GG that can contribute to its probiotic action are cell wall exopolysaccharides (EPS) (Allonsius et al., 2017; Lebeer et al., 2009; Zhang et al., 2016), and the major secreted protein 1 (Msp1), a cell wall hydrolase with anti-pathogenic activity that is also involved in daughter cell separation (Allonsius et al., 2019; Claes et al., 2012).

Isogenic mutants of L. rhamnosus GG have been extensively used to elucidate and validate the role of molecular components involved in cellular physiology and probiotic action. These mutants have played a key role in unravelling the structure and function of SpaCBA pili (Lebeer et al., 2012; Vargas et al., 2015). Likewise, the L. rhamnosus GG knockout mutant in welE encoding the priming glycosyltransferase for a long galactose-rich EPS, showed that the EPS protects L. rhamnosus GG from host factors and might also shield its surface adhesive molecules, including pili (Kiekens et al., 2019; Lebeer et al., 2009, 2011). Additional examples of genetically and phenotypically confirmed L. rhamnosus GG mutants and are listed in Table 1.

Our previous research showed that L. rhamnosus GG can protect primary skin cells from the prominent skin pathogen S. aureus through several mechanisms, including competitive exclusion and pathogen displacement (Mohammedsaeed et al., 2014). Here, we used knock-out mutants of L. rhamnosus GG to further explore the role of probiotic surface molecules and secreted components for probiotic-host and probiotic-pathogen interactions with S. aureus in the skin niche.

\section{Material and methods}

\section{Bacterial strains and culturing conditions}

Bacterial strains used in this study are listed in Table 1. $L$. rhamnosus strains were grown in de Man-Rogosa-Sharpe medium at $37^{\circ} \mathrm{C}$ in static conditions (Spacova et al., 2018). S. aureus was grown at $37^{\circ} \mathrm{C}$ in nutrient broth (Oxoid, Basingstoke, UK) in shaking conditions. Bacteria were harvested after overnight growth by centrifugation at $2,000 \times g$ for $5 \mathrm{~min}$ and washed twice in phosphate-buffered saline (PBS) before use in further assays. Fluorescence induction in L. rhamnosus was performed with nisin from Lactococcus lactis (Sigma-Aldrich, Diegem, Belgium, N5764) as previously described in Spacova et al. (2018).

\section{Cell culturing and adhesion assay}

Normal human epidermal keratinocytes (NHEK) (Promocell, Heidelberg, Germany) were cultured in supplemented Keratinocyte Growth Medium 2 at $37^{\circ} \mathrm{C}$ with $5 \% \mathrm{CO}_{2}$ according to the manufacturer's instructions. For adhesion assays, NHEK cells were grown in 12-well plates until fully confluent. Live bacteria were added to NHEK cells at approximately $10^{6} \mathrm{cfu} /$ well for $S$. aureus and $10^{8} \mathrm{cfu} /$ well for the L. rhamnosus strains, as previously described (Mohammedsaeed et al., 2014). After $1 \mathrm{~h}$ of co-incubation, NHEK cells were washed in PBS, detached using trypsin (Promocell) and serial dilutions in PBS were plated out on either MSA or Baird-Parker (BP) agar (for S. aureus counts) or MRS agar (for L. rhamnosus counts). Percentage of adherent bacteria was calculated and standardised to the L. rhamnosus GG (for L. rhamnosus counts) or S. aureus control well counts.

\section{Fluorescence microscopy}

Fluorescence microscopy assay was performed as previously described (Spacova et al., 2018). Briefly, NHEK cells were grown on glass slides until confluent and fixed in formaldehyde/glutaraldehyde buffer. Fluorescent strains of wild-type L. rhamnosus GG, L. rhamnosus GG spaCBA mutant or wild-type L. rhamnosus GR-1 expressing mCherry were subsequently added to the cells at a concentration of $10^{8} \mathrm{cfu} /$ well, incubated for $1 \mathrm{~h}$ and washed three times with PBS. L. rhamnosus binding was visualised by fluorescence microscopy (Zeiss Axio Imager Z1, Carl Zess AG, Oberkochen, Germany). Three repetitions have been performed for each condition.

\section{Autoaggregation and co-aggregation of bacteria}

A modified quantitative spectrophotometric assay (Collado et al., 2008; Ledder et al., 2008) was implemented to determine autoaggregation of L. rhamnosus strains and co-aggregation with S. aureus. Briefly, L. rhamnosus strains 
Table 1. Bacterial strains used in this study.

\begin{tabular}{|c|c|c|c|}
\hline & Strain name & Phenotype & References \\
\hline \multirow[t]{3}{*}{$\begin{array}{l}\text { Wild-type } \\
\text { strains }\end{array}$} & $\begin{array}{l}\text { Staphylococcus aureus } \\
\text { (clinical isolate) }\end{array}$ & Clinical (skin boil) isolate of $S$. aureus from a human patient & $\begin{array}{l}\text { Gift of Prof. Andrew McBain, } \\
\text { University of Manchester, UK }\end{array}$ \\
\hline & $\begin{array}{l}\text { wt LGG } \\
\text { Lacticaseibacillus rhamnosus GG }\end{array}$ & Wild-type (wt) L. rhamnosus GG ATCC 53103 & $\begin{array}{l}\text { Gorbach, 1996; Segers and } \\
\text { Lebeer, } 2014\end{array}$ \\
\hline & $\begin{array}{l}\text { LGR-1 } \\
\text { L. rhamnosus GR-1 }\end{array}$ & Wild-type (wt) L. rhamnosus GR-1 ATCC 5582 & Reid et al., 2003 \\
\hline \multirow[t]{4}{*}{$\begin{array}{l}\text { Fluorescent } \\
\text { strains }\end{array}$} & S. aureus-GFP & $\begin{array}{l}\text { S. aureus constitutively expressing the green fluorescent } \\
\text { protein (GFP) }\end{array}$ & $\begin{array}{l}\text { Gift of Prof. Alexander Horswill, } \\
\text { University of Colorado } \\
\text { (USA); Malone et al., } 2009\end{array}$ \\
\hline & CMPG11261 & $\begin{array}{l}\text { L. rhamnosus GG expressing the fluorescent mCherry protein } \\
\text { using the nisin-inducible gene expression (NICE) system }\end{array}$ & Spacova et al., 2018 \\
\hline & CMPG11265 & $\begin{array}{l}\text { L. rhamnosus GR-1 expressing the fluorescent mCherry } \\
\text { protein using the nisin-inducible gene expression (NICE) } \\
\text { system }\end{array}$ & Spacova et al., 2018 \\
\hline & CMPG11270 & $\begin{array}{l}\text { L. rhamnosus GG SpaCBA pili mutant expressing the } \\
\text { fluorescent mCherry protein using the nisin-inducible gene } \\
\text { expression (NICE) system }\end{array}$ & Spacova et al., 2018 \\
\hline \multirow[t]{10}{*}{$\begin{array}{l}\text { L. rhamnosus } \\
\text { mutants }\end{array}$} & $\begin{array}{l}\text { LGG spaCBA } \\
\text { L. rhamnosus CMPG5357 }\end{array}$ & $\begin{array}{l}\text { SpaCBA pili mutant; impaired biofilm formation and adhesion } \\
\text { to human intestinal epithelial cells and macrophages due to } \\
\text { lack of pili }\end{array}$ & $\begin{array}{l}\text { Kiekens et al., 2019; Lebeer et } \\
\text { al., 2012; Vargas Garcia et } \\
\text { al., } 2015\end{array}$ \\
\hline & $\begin{array}{l}\text { LGG welE } \\
\text { L. rhamnosus CMPG5351 }\end{array}$ & $\begin{array}{l}\text { Cell wall exopolysaccharides (EPS) mutant, diminished EPS } \\
\text { layer; pili and potentially other cell surface adhesins are more } \\
\text { exposed, increased adhesion to human intestinal epithelial } \\
\text { cells and murine macrophages }\end{array}$ & $\begin{array}{l}\text { Lebeer et al., 2009, 2011; } \\
\text { Kiekens et al., } 2019\end{array}$ \\
\hline & $\begin{array}{l}\text { LGG welE/spaC } \\
\text { L. rhamnosus CMPG5365 }\end{array}$ & $\begin{array}{l}\text { Cell wall EPS and SpaC pili subunit mutant; deficient in } \\
\text { adhesion to human intestinal epithelial cells and biofilm } \\
\text { formation }\end{array}$ & Lebeer et al., 2012 \\
\hline & $\begin{array}{l}\text { LGG msp1 } \\
\text { L. rhamnosus CMPG10200 }\end{array}$ & $\begin{array}{l}\text { Major secreted protein } 1 \text { (Msp1) mutant producing truncated } \\
\text { version of Msp1; impaired in cell separation during cell } \\
\text { division }\end{array}$ & Lebeer et al., 2012 \\
\hline & $\begin{array}{l}\text { LGG Ilp1 } \\
\text { L. rhamnosus CMPG10701 }\end{array}$ & $\begin{array}{l}\text { Lectin-like protein } 1 \text { mutant; reduced adhesion to human } \\
\text { intestinal epithelial cells }\end{array}$ & Petrova et al., 2016 \\
\hline & $\begin{array}{l}\text { LGG Ilp2 } \\
\text { L. rhamnosus CMPG10706 }\end{array}$ & $\begin{array}{l}\text { Lectin-like protein } 2 \text { mutant; reduced adhesion to human } \\
\text { intestinal and vaginal epithelial cells, increased biofilm } \\
\text { formation }\end{array}$ & Petrova et al., 2016 \\
\hline & $\begin{array}{l}\text { LGG Ilp1/2 } \\
\text { L. rhamnosus CMPG10707 }\end{array}$ & $\begin{array}{l}\text { Lectin-like proteins } 1 \text { and } 2 \text { mutant; reduced adhesion to } \\
\text { human intestinal and vaginal epithelial cells }\end{array}$ & Petrova et al., 2016 \\
\hline & $\begin{array}{l}\text { LGG mabA } \\
\text { L. rhamnosus CMPG5230 }\end{array}$ & $\begin{array}{l}\text { Modulator of adhesion and biofilm formation (MabA) mutant; } \\
\text { reduced adhesion to human intestinal epithelial cells and } \\
\text { biofilm formation }\end{array}$ & Perea Vélez et al., 2010 \\
\hline & $\begin{array}{l}\text { LGG adhA } \\
\text { L. rhamnosus CMPG5355 }\end{array}$ & Putative adhesion (AdhA) exoprotein mutant & Lebeer et al., 2012 \\
\hline & $\begin{array}{l}\text { LGG secX } \\
\text { L. rhamnosus CMPG10816 }\end{array}$ & $\begin{array}{l}\text { Mutant in SecX accessory transport system for glycosylated } \\
\text { proteins }\end{array}$ & Tytgat et al., 2015 \\
\hline
\end{tabular}

as such or mixed with $S$. aureus were resuspended in PBS to a concentration of $2 \times 10^{8} \mathrm{cfu} / \mathrm{ml}$. After incubation at room temperature, optical density in cuvettes was recorded at 600 $\mathrm{nm}\left(\mathrm{OD}_{600}\right)$. The following equation was used to determine the percentages of auto- or co-aggregation:
Aggregation percentage $=$

$$
\frac{\left.\mathrm{OD}_{600} \text { (before incubation }\right)-\mathrm{OD}_{600}(\text { after incubation })}{\mathrm{OD}_{600}(\text { before incubation })} \times 100
$$




\section{Keratinocyte viability assay}

Keratinocyte viability in co-culture with bacteria was determined as previously described (Prince et al., 2012). Briefly, live bacteria were added to fully confluent NHEK cells in 12-well plates at approximately $10^{6} \mathrm{cfu} /$ well for S. aureus and $10^{8} \mathrm{cfu} /$ well for the L. rhamnosus strains, as previously described (Mohammedsaeed et al., 2014). After $24 \mathrm{~h}$ of co-incubation at $37^{\circ} \mathrm{C}$ and $5 \% \mathrm{CO}_{2}$, NHEK cells were washed in PBS and detached using trypsin. Cell viability was determined by Trypan blue exclusion assay was implemented to assess NHEK viability as described by Strober (1997).

\section{Time-course analysis of Staphylococcus aureus growth with spent culture supernatants or live Lacticaseibacillus rhamnosus}

Spent culture supernatant was obtained from overnight cultures of $L$. rhamnosus in MRS by centrifugation (15 min, $\left.4,000 \times g, 4^{\circ} \mathrm{C}\right)$ with subsequent filter sterilisation $(0.20 \mu \mathrm{m}$, VWR, Leuven, Belgium) and $\mathrm{pH}$ measurement (MettlerToledo AG, Zaventem, Belgium). S. aureus growth inhibition by spent culture supernatant or live L. rhamnosus was performed as previously described (Van den Broeck et al., 2018). Briefly, overnight cultures of wt or GFP-producing $S$. aureus were diluted 1:100 in Mueller Hinton (MH) medium in a 96-well plate, and either live L. rhamnosus at the same dilution, or L. rhamnosus supernatant at 1:5 dilution were added to the wells. Growth of S. aureus was assessed over the course of 19 to $48 \mathrm{~h}$, either by optical density (OD) at $600 \mathrm{~nm}\left(\mathrm{OD}_{600}\right)$, or GFP fluorescence measurement at $488 / 510 \mathrm{~nm}$ in a multi-mode microplate reader (Synergy $^{\mathrm{mm}}$ HTX, BioTek, Winooski, VT, USA).

\section{NF-kB pathway activation as a result of Toll-like receptor stimulation in a THP-1 reporter cell line}

THP1-Dual ${ }^{\mathrm{Tt}}$ cells (InvivoGen, San Diego, CA, USA) were maintained according to manufacturer's instructions in growth medium containing RPMI 1640, 2 mM L-glutamine, $25 \mathrm{mM}$ HEPES, 10\% heat-inactivated foetal bovine serum, $100 \mu \mathrm{g} / \mathrm{ml}$ Normocin ${ }^{\mathrm{Tx}}$ and Pen-Strep (100 U/ml-100 $\mu \mathrm{g} /$ $\mathrm{ml})$. For assessment of nuclear factor kappa beta (NF-kB) pathway activation as a result of Toll-like receptor (TLR) stimulation, $S$. aureus was added to THP- 1 at $10^{6} \mathrm{cfu} /$ $\mathrm{ml}$, while L. rhamnosus strains were added at $10^{7} \mathrm{cfu} / \mathrm{ml}$, and co-incubated for $24 \mathrm{~h}$ at $37^{\circ} \mathrm{C}$ and $5 \% \mathrm{CO}_{2}$. NF-kBSEAP activity was measured at $405 \mathrm{~nm}$ according to the manufacturer's instructions after addition of a p-nitrophenyl phosphate (pNPP) solution.

\section{Statistical analysis}

Normal distribution of data was evaluated using ShapiroWilk and Kolmogorov-Smirnov normality tests. One- sample $t$ test (for parametric data) or the Wilcoxon Signed Rank Test (for non-parametric data) were performed to statistically compare data to control values corresponding to $100 \%$. In other cases, either an unpaired t test (for parametric data) or Mann-Whitney U (for non-parametric data) test was used for pairwise comparisons, and one-way or two-way ANOVA was used for comparisons between several groups. For combining adhesion experiments data from different 12-well plates, values obtained in control wells (either S. aureus or wt L. rhamnosus GG) in each plate were set to $100 \%$, and other tested conditions were percentage scaled to control wells values. Figure 6 was created with BioRender.com.

\section{Results}

\section{Presence of SpaCBA pili is crucial for Lacticaseibacillus rhamnosus $\mathrm{GG}$ adhesion to keratinocytes}

First, we aimed to identify key cell surface molecules involved in efficient binding of L. rhamnosus GG to human epidermal keratinocytes in vitro. We screened a selection of previously validated $L$. rhamnosus GG knock-out mutants in probiotic and adhesion-related molecules (Table 1 ) and compared their adhesion to wild-type (wt) L. rhamnosus GG (Figure 1A). The mutants used in this study have been extensively characterised in previous studies as to their mutant phenotype and molecular and cellular interactions (Table 1). However, their interactions with S. aureus or effects on keratinocytes have not yet been described.

Adhesion decreased significantly in spaCBA and welE/spaC mutants ( 24 times in LGG spaCBA and $\sim 4$ times in LGG welE/spaC, $P<0.01$ ) and increased, albeit non-significantly, in two of the welE mutants (LGG welE, $P=0.0534$ and LGG welE/msp1) compared to wt L. rhamnosus GG. Of note, adhesion was significantly higher $(P<0.01)$ for the $L$. rhamnosus GG welE/spaC mutant compared to $L$. rhamnosus GG spaCBA mutant and significantly lower $(P<0.01)$ compared to the L. rhamnosus GG welE mutant. Adhesion was not significantly affected in L. rhamnosus GG msp1, mabA, adhA, llp1, llp2 and $l l p 1 / 2$ mutants, and the llp1, llp2 and llp1/2 mutants were omitted from further analysis. A closely related urogenital probiotic strain L. rhamnosus GR-1 lacking SpaCBA pili was used for comparison and was found to have approximately 12 times lower adhesion levels to keratinocytes $(P<0.0001)$ compared to L. rhamnosus GG (Figure 1A).

To further validate these results, binding of fluorescent $L$. rhamnosus GG (strain CMPG11261), L. rhamnosus GG spaCBA mutant (strain CMPG11270) and L. rhamnosus GR-1 (strain CMPG11265) to keratinocytes was visualised by fluorescence microscopy (Figure 1B). The images confirmed that the fluorescent $L$. rhamnosus GG spaCBA knock-out mutant and L. rhamnosus GR-1 do not efficiently 


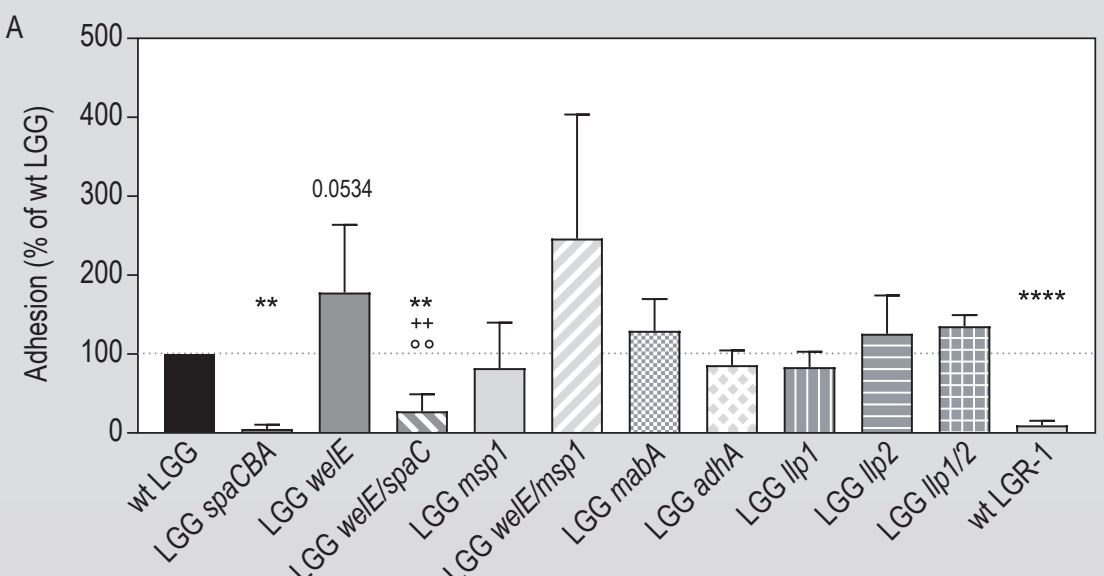

B CMPG11261

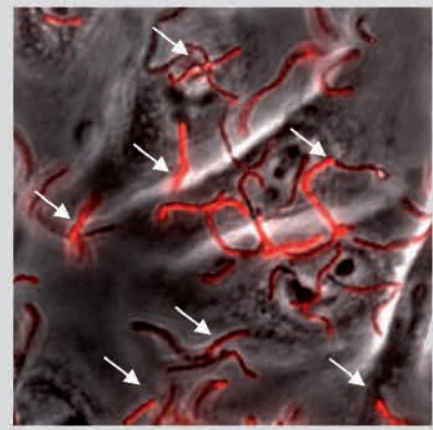

CMPG11270

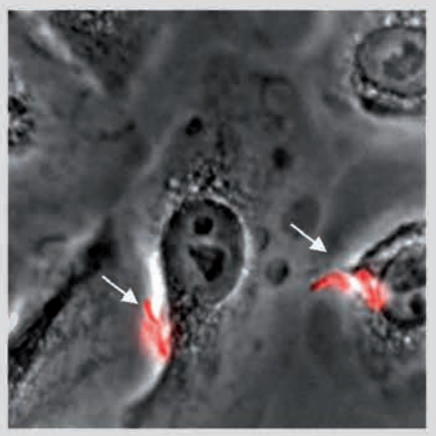

CMPG11265

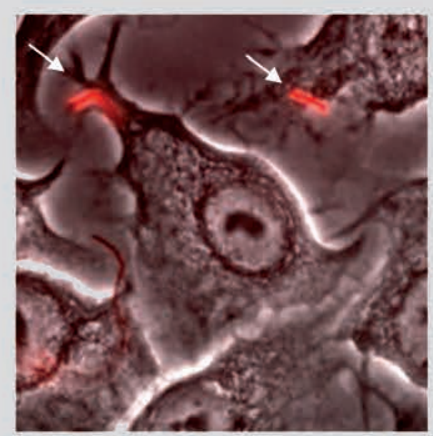

Figure 1. (A) Adhesion of Lacticaseibacillus rhamnosus GG knock-out mutants and L. rhamnosus GR-1 to human keratinocytes compared to wt $L$. rhamnosus GG. (B) Adhesion of fluorescent $L$. rhamnosus GG (strain CMPG11261) to human keratinocytes compared to fluorescent $L$. rhamnosus GG spaCBA mutant (strain CMPG11270) and L. rhamnosus GR-1 (strain CMPG11265) visualised by fluorescence microscopy. Results are depicted as mean \pm standard deviation $(n=3-9)$. Data is expressed as percentages of the wild-type L. rhamnosus GG (wt LGG) adhesion percentage, which is set to $100 \%$ (dotted line). ${ }^{* *} P<0.01$ and ${ }^{* * * *} P<0.0001$ compared to $100 \%$ representing adhesion of wt LGG; $++P<0.01$ compared to LGG spaCBA; ${ }^{\circ 0} P<0.01$ compared to LGG welE. Strains are labelled according to Table 1.

adhere to human keratinocytes, in contrast to the wt $L$. rhamnosus GG that binds to keratinocytes in high numbers.

\section{Prevention of Staphylococcus aureus adhesion is decreased in Lacticaseibacillus rhamnosus GG spaCBA mutants}

L. rhamnosus GG, L. rhamnosus GG knock-out mutants and L. rhamnosus GR-1 were further tested for their ability to inhibit $S$. aureus adhesion to human keratinocytes. $L$. rhamnosus GG significantly inhibited S. aureus adhesion, in contrast to L. rhamnosus GR-1 (Figure 2A). The spaCBA and welE/spaC mutants showed significantly less inhibition of $S$. aureus adhesion (Figure $2 \mathrm{~A}, P<0.01$ and $P<0.05$, respectively). Interestingly, the $L$. rhamnosus GG msp1 knock-out mutant known to form aggregates due to a defect in daughter cell separation demonstrated a significantly stronger inhibition of $S$. aureus adhesion compared to wt L. rhamnosus GG $(P<0.05)$. L. rhamnosus $\mathrm{GG}$ mabA and adh $A$ knock-out mutations did not significantly affect $L$. rhamnosus GG adhesion to keratinocytes or prevention of $S$. aureus adhesion and were omitted from further analysis.

Adhesion to keratinocytes in co-cultures of fluorescent $S$. aureus with either L. rhamnosus GG, L. rhamnosus GG spaCBA mutant or L. rhamnosus GR-1 was visualised by fluorescence microscopy (Figure 2B). S. aureus cells were less adherent to regions of keratinocytes densely occupied by $L$. rhamnosus GG, while no regions densely occupied with $L$. rhamnosus GG spaCBA knock-out mutant and $L$. rhamnosus GR-1 were observed.

\section{Lacticaseibacillus rhamnosus GG co-aggregates with Staphylococcus aureus, which is less pronounced in the spaCBA and welE mutants}

Next, we analysed L. rhamnosus GG, L. rhamnosus GR-1 and a selection of L. rhamnosus GG knock-out mutants to assess autoaggregation, and co-aggregation with $S$. aureus. Wt L. rhamnosus GG showed more pronounced 
A

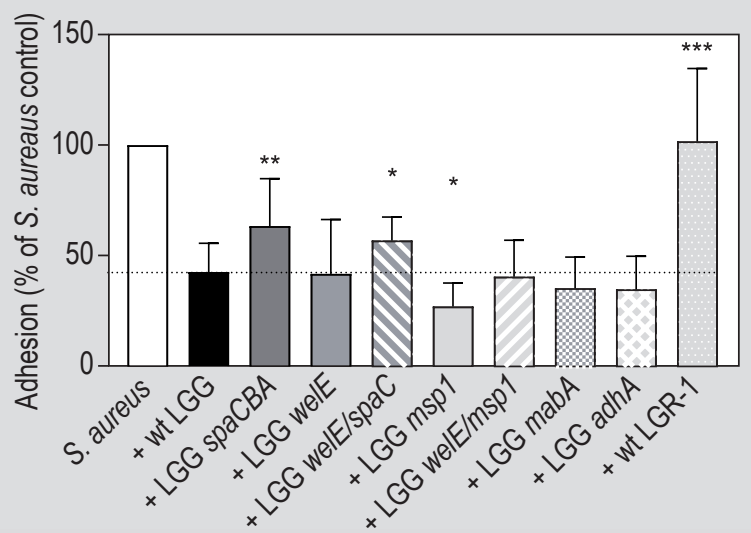

B

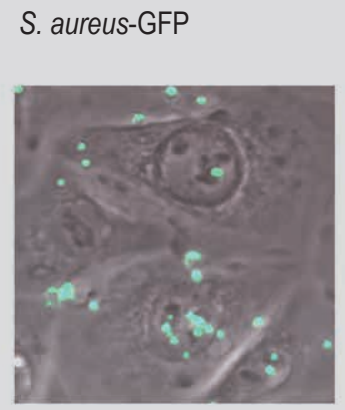

S. aureus-GFP+ CMPG11270

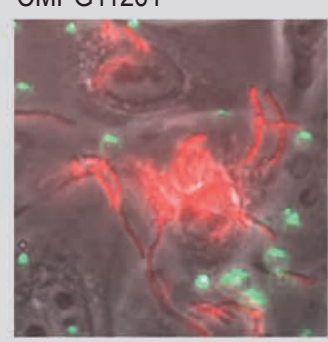

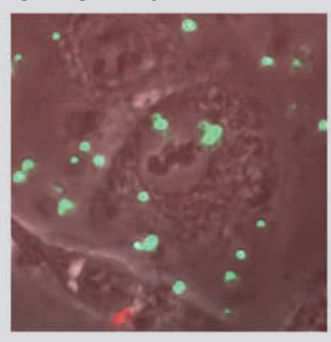

S. aureus-GFP+ CMPG112765

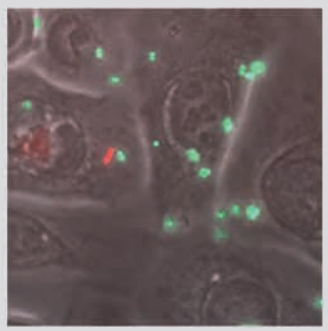

Figure 2. (A) Prevention of Staphylococcus aureus adhesion to human keratinocytes by Lacticaseibacillus rhamnosus GG knockout mutants and $L$. rhamnosus GR-1 compared to $L$. rhamnosus GG; (B) Adhesion of fluorescent $S$. aureus-GFP(in green) in the presence of fluorescent $L$. rhamnosus GG (CMPG11261), fluorescent $L$. rhamnosus GG spaCBA mutant (CMPG11270) or fluorescent L. rhamnosus GR-1 (CMPG11265) (in red) to human keratinocytes visualised by fluorescence microscopy. Keratinocytes were incubated either with $S$. aureus alone ( $S$. aureus condition) or with different $L$. rhamnosus strains (all + conditions). Results are depicted as mean \pm standard deviation, and expressed as percentages of adherent $S$. aureus cfu counts in control wells ( $S$. aureus with no $L$. rhamnosus added) ( $n=5-13)$. Dotted line indicates the result obtained for the $S$. aureus with wild-type $L$. rhamnosus GG condition. ${ }^{*} P<0.05,{ }^{* *} P<0.01$ and ${ }^{* * *} P<0.001$ when compared to wt $L G G$ condition. Strains are labelled according to Table 1.

co-aggregation with $S$. aureus compared to L. rhamnosus GG spaCBA $(P<0.001)$ and welE mutants (not significant), and L. rhamnosus GR-1 $(P<0.0001)$ (Figure 3A). The $L$. rhamnosus GG msp1 $(P<0.0001)$ and L. rhamnosus GG welE/msp $1(P<0.001)$ mutants, and L. rhamnosus GR-1 $(P<0.0001)$, had a significantly stronger autoaggregative phenotype compared to wt $L$. rhamnosus GG (Figure 3B).

\section{Lacticaseibacillus rhamnosus GG spaCBA and welE/} msp1 mutants are less efficient in improving keratinocyte viability in long-term co-culture with Staphylococcus aureus

In addition to assessing probiotic-pathogen interactions over short periods of time, we further aimed to elucidate the effects of long-term co-incubation of L. rhamnosus GG and its knock-out mutants on S. aureus infection of human keratinocytes. Hereto, S. aureus was co-incubated with primary human keratinocytes for $24 \mathrm{~h}$, alone or in combination with L. rhamnosus GG, a selection of
L. rhamnosus GG knock-out mutants or L. rhamnosus GR-1 (Figure 4A). All tested L. rhamnosus strains except for L. rhamnosus GG spaCBA and welE/msp1 mutants significantly improved keratinocyte viability in a $24 \mathrm{~h}$ coculture with S. aureus (Figure 4A).

\section{Secreted factors of Lacticaseibacillus rhamnosus $\mathbf{G G}$ and GR-1 contributing to medium acidification inhibit long- term Staphylococcus aureus growth}

To explore additional anti-pathogenic mechanisms of L. rhamnosus GG during long-term co-incubation with pathogens, we investigated the potential role of secreted probiotic molecules that inhibit $S$. aureus growth. Hereto, S. aureus was co-incubated for $48 \mathrm{~h}$ with spent culture supernatant of L. rhamnosus GG, L. rhamnosus GG knockout mutants or L. rhamnosus GR-1 (Figure 4). Supernatants of L. rhamnosus GG and L. rhamnosus GR-1 drastically inhibited S. aureus growth (Figure 4B). However, when the supernatant $\mathrm{pH}$ was adjusted to 7.4, the inhibition was 

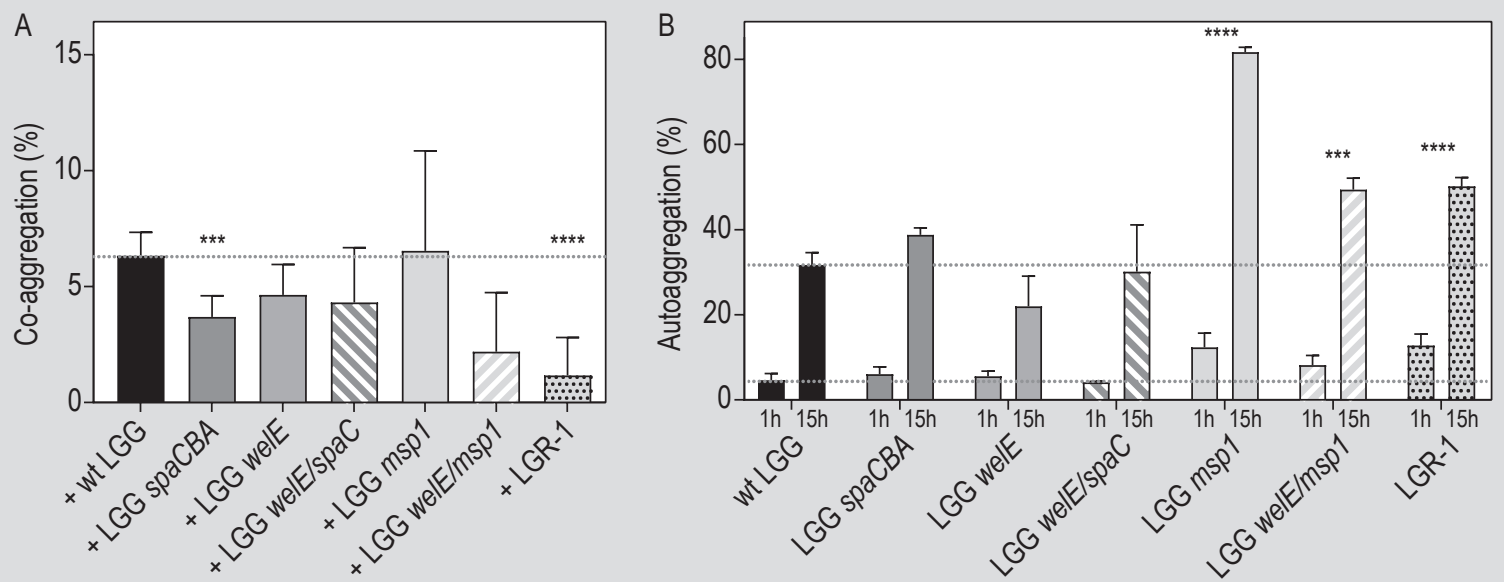

Figure 3. Co-aggregation with Staphylococcus aureus after $1 \mathrm{~h}(\mathrm{~A})$ and autoaggregation after $1 \mathrm{~h}$ and $15 \mathrm{~h}$ (B) for Lacticaseibacillus rhamnosus $G G$, $L$. rhamnosus $G$ G knock-out mutants and $L$. rhamnosus $G R-1$. S. aureus was incubated with different $L$. rhamnosus strains (all + conditions) (A), or bacterial strains were incubated alone (B). Representative results are depicted as mean \pm standard deviation, and expressed as percentages of co- or autoaggregation $(n=3)$. Dotted line indicates the results obtained for wild-type L. rhamnosus GG. ${ }^{* * *} P<0.001$ and ${ }^{* * \star *} P<0.0001$ when compared to wt $L G G$ condition. Strains are labelled according to Table 1.

A
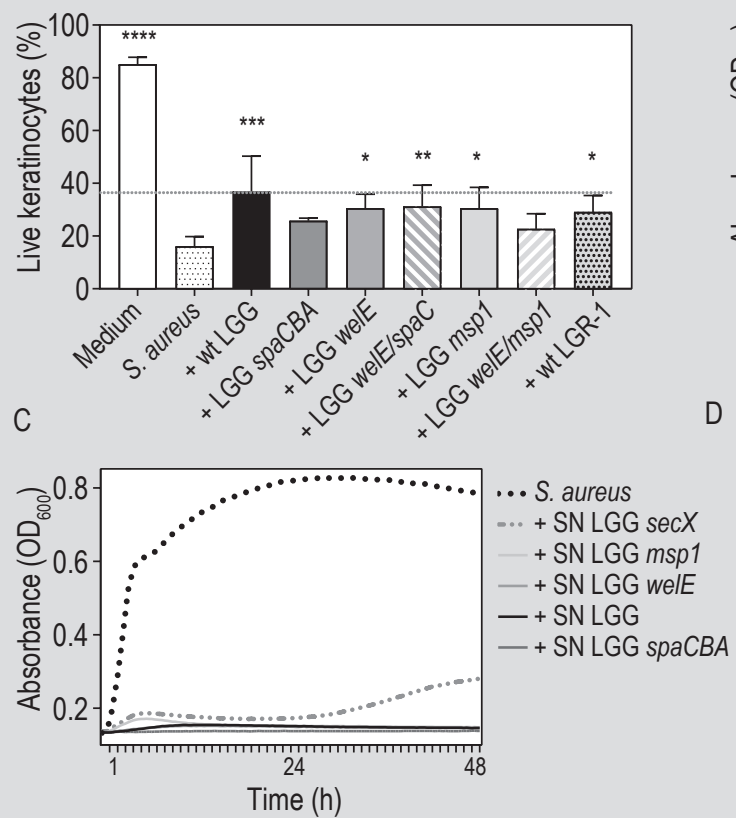

B

D
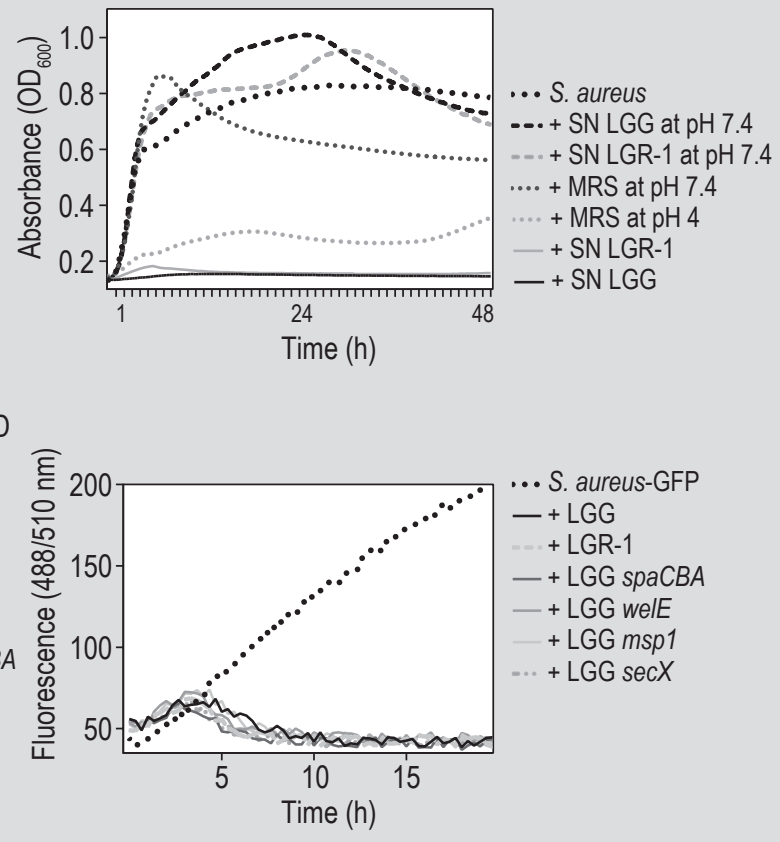

Figure 4. Effects of long-term co-incubation of Lacticaseibacillus rhamnosus with Staphylococcus aureus. (A) Improvement of keratinocyte viability by wild-type $L$. rhamnosus GG mutants and $L$. rhamnosus GR-1 after 24 h co-incubation with $S$. aureus. Representative results are depicted as mean \pm standard deviation, and expressed as percentages of live cells from the total number of counted cells ( $n=3-4)$. Dotted line indicates the results obtained for cells co-incubated with $S$. aureus and wild-type L. rhamnosus GG. * $P<0.05$, ** $P<0.01$, ${ }^{* *} P<0.001$ and ${ }^{* * *} P<0.0001$ when compared to $S$. aureus. S. aureus growth kinetics with (B-C) supernatants (SN) of L. rhamnosus GG mutants and L. rhamnosus GR-1, or (D) live L. rhamnosus over the course of 19-48 $h$ depicted as means per time point $(n=3)$. Strains are labelled according to Table 1. 
completely abrogated. Likewise, when the MRS medium control $\mathrm{pH}$ was adjusted to 4 (approximately equal to the $\mathrm{pH}$ of $L$. rhamnosus GG and L. rhamnosus GR-1 supernatants, Table 2), S. aureus growth was inhibited, although not as strongly as with Lacticaseibacillus supernatants. Knock-out mutations in L. rhamnosus GG had no significant effects on the ability of live lactobacilli or their supernatants to inhibit S. aureus growth. L. rhamnosus GG secX mutant impaired in secretion of glycosylated protein compounds was additionally included in this analysis, and there was a tendency towards a less pronounced S. aureus growth inhibition by the supernatant of this strain (Figure 4C). Coculturing of an alternative fluorescent $S$. aureus strain with live L. rhamnosus GG, L. rhamnosus GG knock-out mutants or $L$. rhamnosus GR-1 likewise completely inhibited $S$. aureus growth (Figure 4D).

\section{Lacticaseibacillus rhamnosus reduces inflammatory cascade activation by Staphylococcus aureus when live and stimulates host Toll-like receptors}

Finally, we aimed to explore the degree of host TLR stimulation in co-culture with live $S$. aureus, alone or in combination with $L$. rhamnosus. Hereto, we implemented a THP-1 monocyte reporter cell line that allows estimation of NF- $\mathrm{kB}$ activation in response to TLR agonists.

Co-incubation with all live L. rhamnosus strains except for $L$. rhamnosus GG welE/msp1 mutant reduced NF-кB pathway activation by $S$. aureus in these monocytic human cells (Figure 5A). Significant effects of $L$. rhamnosus GG knock-out mutations were detected: live L. rhamnosus GG spaCBA, msp1 and welE/spaC mutants lowered TLR activation by $S$. aureus in these cells more efficiently, compared to wt $L$. rhamnosus GG (Figure 5A). The $L$. rhamnosus GG welE and L. rhamnosus GG welE/msp1 mutants increased $S$. aureus-related TLR activation in the THP-1 reporter cells when compared to wt $L$. rhamnosus GG (Figure 5A). When UV-inactivated $S$. aureus and $L$. rhamnosus were combined, all L. rhamnosus strains rather showed a cumulative immunostimulatory effect with $S$. aureus (Figure 5B), in contrast to when live bacteria were used. This immunostimulatory effect was more pronounced for the L. rhamnosus GG welE mutants in welE, welE/spaC and welE/msp1 (Figure 5B). Similarly, when UV-inactivated L. rhamnosus was co-incubated with live S. aureus, no reduction in TLR activation was observed (Figure 5C).
TLR activation by UV-inactivated S. aureus (Figure 5D) in the THP-1 reporter cells was much lower compared to live S. aureus. Live and UV-inactivated L. rhamnosus GG, $L$. rhamnosus GG mutants and L. rhamnosus GR-1 alone were also capable of weak TLR induction (Figure 5D), although at a significantly lower degree than $S$. aureus. The pattern observed for TLR induction by live L. rhamnosus (Figure 5D, 'live') was generally similar to that induced by UVinactivated L. rhamnosus (Figure 5D, 'UV'). Intriguingly, only L. rhamnosus GG welE/msp1 induced a much stronger TLR-facilitated response when added live. Live and UVinactivated L. rhamnosus GG spaCBA mutant caused less TLR receptor activation compared to wt $L$. rhamnosus GG (Figure 5C) in these specific THP-1 derived reporter cells, while all other tested knock-out mutants appear to be more immunostimulatory compared to wt $L$. rhamnosus GG.

\section{Discussion}

Cell surface and secreted molecules of probiotic bacteria play a crucial role in their direct and indirect interaction with the surrounding environment. In particular, efficient adherence of probiotic L. rhamnosus GG to host cells mediated by these molecules contributes to longer host colonisation and competitive exclusion of pathogens (Lebeer et al., 2018a; Segers and Lebeer, 2014). In this study, we implemented L. rhamnosus GG, L. rhamnosus GR-1 and a range of L. rhamnosus GG knock-out mutants in cell surface and secreted molecules to identify the L. rhamnosus GG molecules involved in its probiotic mechanisms of action against a clinical S. aureus isolate in co-culture with human skin cells.

Our data suggests an important contribution of SpaCBA pili to pathogen exclusion of $S$. aureus by $L$. rhamnosus GG. Specifically, L. rhamnosus GR-1 and L. rhamnosus GG mutants lacking SpaCBA pili or the SpaC pilin were significantly less efficient in prevention of $S$. aureus adhesion to keratinocytes. Numerous studies demonstrated inhibition of pathogen adhesion to host cells by lactobacilli in co-cultures with intestinal (Burkholder and Bhunia, 2009) or vaginal (Zárate and Nader-Macias, 2006) cells, either through steric hindrance or competition for receptors and specific adhesion sites. Interestingly, in contrast to our data, L. rhamnosus GR-1 was previously shown to displace and prevent the binding of $S$. aureus to female uroepithelial cells (Reid et al., 1995), possibly highlighting

Table 2. pH of the corresponding Lacticaseibacillus rhamnosus supernatants. ${ }^{1}$

\begin{tabular}{|c|c|c|c|c|c|c|}
\hline Strain & LGG & LGG spaCBA & LGG welE & LGG msp1 & LGG sec $X$ & LGR-1 \\
\hline $\mathrm{pH}$ & 3.84 & 3.81 & 3.91 & 4.08 & 4.18 & 4.08 \\
\hline
\end{tabular}



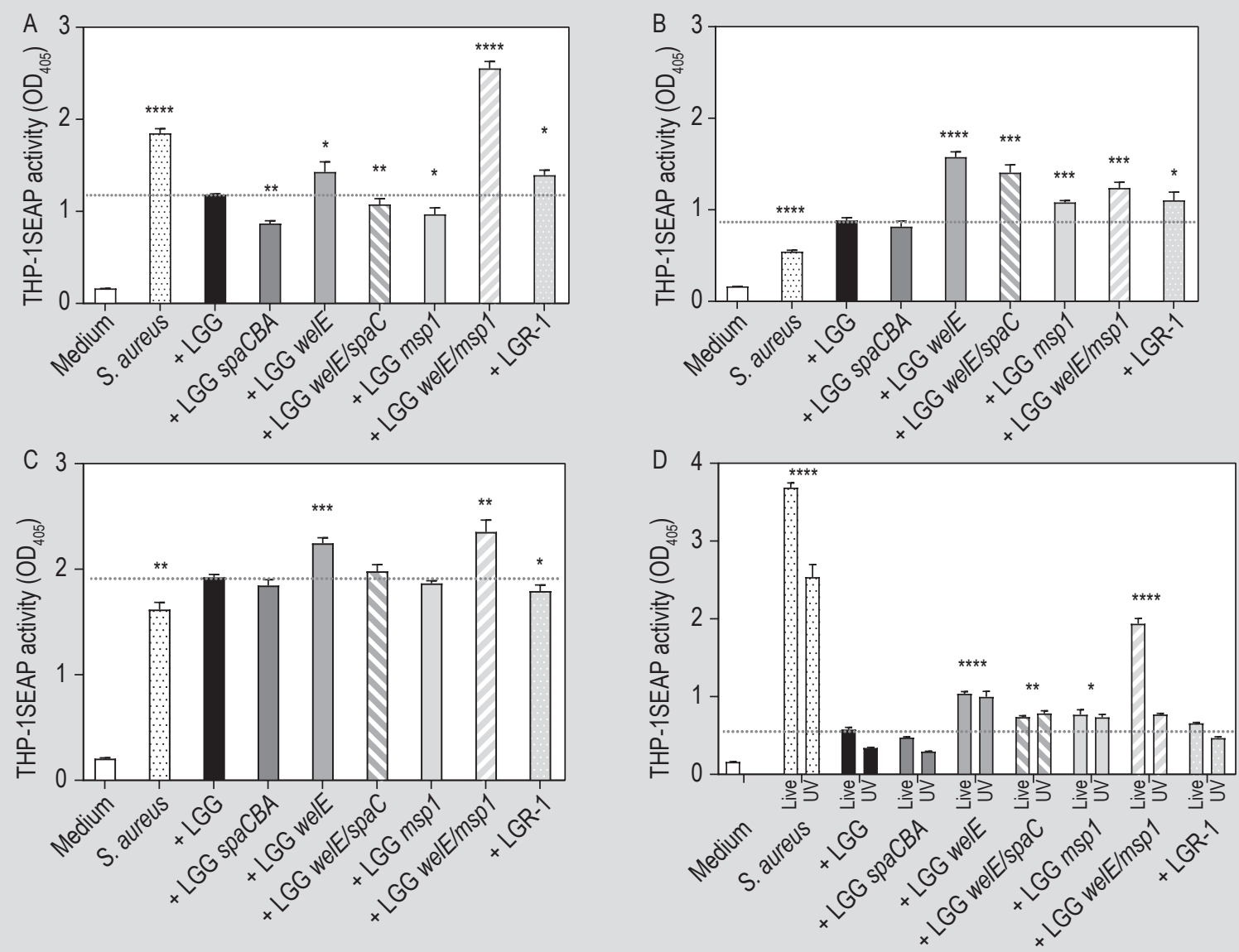

Figure 5. Toll-like (TLR) receptor activation in THP-1 reporter cells co-cultured with (A) live Staphylococcus aureus together with live Lacticaseibacillus rhamnosus; (B) UV-inactivated S. aureus together with UV-inactivated L. rhamnosus; (C) Live S. aureus together with UV-inactivated $L$. rhamnosus; (D) Live or UV-inactivated S. aureus or $L$. rhamnosus alone. Representative results are depicted as mean \pm standard deviation bars, and expressed as $\mathrm{OD}_{405}$ values representing NF-KB-induced SEAP activity as a result of TLR activation assessed with $\mathrm{PNPP}(\mathrm{n}=3)$. ${ }^{*} P<0.05$, ${ }^{* *} P<0.01,{ }^{* * *} P<0.001$ and ${ }^{* * * *} P<0.0001$ when compared to either $S$. aureus with wt LGG, or wt LGG alone conditions. Strains are labelled according to Table 1.

the body niche-dependent differences in probiotic-pathogen and host interactions. Furthermore, our results suggest that the observed probiotic-pathogen interactions are complex, and not determined solely by the presence of SpaCBA pili or the adhesion efficacy of L. rhamnosus GG mutants to keratinocytes.

We showed that the presence of SpaCBA pili is also crucial for efficient adhesion of L. rhamnosus to keratinocytes. Previous studies suggest that pili of gram-positive bacteria govern tissue tropism - for example, the SpaB and $\mathrm{SpaC}$ pilins of Corynebacterium diphtheriae facilitate its specific adherence to human pharyngeal epithelial cells (Mandlik et al., 2007). The importance of SpaCBA pili was demonstrated for adhesion to Caco-2 intestinal epithelial cells (Lebeer et al., 2012), murine macrophages (Vargas Garcia et al., 2015) and intestinal mucus (Von Ossowski et al., 2010), and our findings also suggest their key role for skin probiotics. In addition, the L. rhamnosus GG welE mutant impaired in the EPS layer formation showed higher adhesion to keratinocytes, possibly as a result of higher exposure of pili (Burgain et al., 2014; Lebeer et al., 2009; Vargas-Garcia et al., 2015). The results from double mutants impaired in both EPS and SpaC production suggest that cell surface molecules masked by EPS other than SpaCBA might be additionally involved. These surface adhesion molecules potentially include adhesive LPXTG- and WxLanchored cell wall proteins of $L$. rhamnosus GG (Perea Vélez et al., 2010; Petrova et al., 2016; Von Ossowski et al., 2010). While previous studies identified modulator of adhesion and biofilm formation (MabA) (Perea Vélez et al., 2010) and Lectin-like proteins 1 and 2 (Llp1 and Llp2) (Petrova et al., 2016) as L. rhamnosus GG adhesion factors to vaginal and/or intestinal epithelial cells, mutations in the corresponding genes did not significantly alter $L$. rhamnosus GG adhesion to keratinocytes in our experimental set- 
up. Further research with knock-out mutants, blocking antibodies and cell wall fractions of L. rhamnosus GG is required to elucidate the additional factors contributing to L. rhamnosus GG adhesion to skin cells besides SpaCBA pili.

Probiotic-pathogen co-aggregation is another property of L. rhamnosus GG that can help capture free pathogenic bacteria and prevent their adhesion to host cells (Collado et al., 2008). Additionally, probiotic autoaggregation can lead to more efficient competition with pathogens for host adhesion sites. In our experimental set-up, L. rhamnosus GG demonstrates significantly higher co-aggregation with S. aureus compared to L. rhamnosus GR-1, with coaggregation percentages in line with previously reported data (Collado et al., 2008). Our results with L. rhamnosus GG knock-out mutants imply a role of SpaCBA pili, and to a lesser degree EPS, for binding with $S$. aureus. While the effects of each of these molecules on co-aggregation might be considered relatively small in our study, in combination with other cell surface compounds they could contribute to the multifactorial action of $L$. rhamnosus GG. The involvement of EPS would not be surprising, as lectincarbohydrate interactions have previously been suggested to play a role in co-aggregation of L. rhamnosus GG with oral, intestinal and vaginal pathogens (Allonsius et al., 2017; Ledder et al., 2008). Interestingly, in our study $L$. rhamnosus GR-1 demonstrated higher autoaggregation values compared to wt L. rhamnosus GG, despite its inferior ability to co-aggregate with $S$. aureus. Previous research in L. rhamnosus showed a correlation between auto- and coaggregative properties of probiotics (Collado et al., 2008). Nevertheless, our data demonstrates that autoaggregation is not always a predictor for co-aggregation with pathogens, which can be explained by the involvement of different surface molecules during auto- and co-aggregation with other bacterial strains.

All tested live and UV-inactivated L. rhamnosus strains were significantly less immunostimulatory than $S$. aureus in monocyte reporter cells, which is in line with their nonpathogenic status. Additionally, host TLR receptor activation by the pathogen $S$. aureus and probiotic lactobacilli are likely different (Kim et al., 2007), and would therefore lead to differential induction of immune signalling cascades in the host. The key role of cell surface molecules in probiotichost cell interactions was emphasised by the ability of UV-inactivated L. rhamnosus to stimulate TLR activation in host cells. Impairment in EPS formation, a clumping phenotype due to mutated Msp1, or a combination thereof, resulted in more pronounced TLR activation. Previous studies demonstrated that EPS can serve as a protective shield against host factors (Lebeer et al., 2011) and mask bacterial surface structures of L. rhamnosus GG, such as lipoteichoic acid (LTA) that interacts with TLR2/6 (Claes et al., 2012). This suggests that increased and closer contact of bacterial surface molecules with host cells due to highly autoaggregative and adhesive probiotic phenotypes could be more immunostimulatory for monocytes/immune cells, although this requires further substantiation.

Other probiotic mechanisms of action in addition to adhesion prevention might become important when $S$. aureus infection of host cells is performed over a longer time period. Live but not UV-inactivated intact L. rhamnosus was able to downregulate the long-term inflammatory response to $S$. aureus in monocytes. Both L. rhamnosus GR-1 and the L. rhamnosus GG mutant lacking SpaCBA pili still partially increased the viability of keratinocytes in long-term co-culture with S. aureus, despite their inability to prevent $S$. aureus adhesion. Finally, in our experimental set-up, pro-inflammatory responses induced by S. aureus in monocytes were highly dependent on its viability and possibly infectivity, which might be influenced by secreted L. rhamnosus molecules.

In fact, our data points to the key role of secreted $L$. rhamnosus metabolites with $\mathrm{pH}$-reducing properties in preventing $S$. aureus infection of keratinocytes. As live L. rhamnosus was shown to inhibit $S$. aureus growth, the reduction in immunostimulation of monocytes might be partly explained by $S$. aureus growth inhibition as a result of $\mathrm{pH}$ decrease. We indeed further demonstrated that the growth inhibition of S. aureus by L. rhamnosus was dependent on $\mathrm{pH}$ reduction of the culture medium. Although a minor contribution of secreted glycosylated components could be suggested, the inhibitory effect of L. rhamnosus GG on long-term S. aureus growth is likely linked to lactic acid production. This contributes to previous data on the key role of lactic acid for antipathogenic activity of L. rhamnosus GG against bacterial pathogens Salmonella Typhimurium (De Keersmaecker et al., 2006) and Moraxella catarrhalis (Van den Broek et al., 2018).

Healthy skin surface $\mathrm{pH}$ is estimated to be 4.7 on average (Lambers et al., 2006) and lactic acid contributing to an acidic $\mathrm{pH}$ is already used for dermatological applications, for example to improve keratoses and acne (Tang et al., 2018). While the capacity for lactic acid production by lactobacilli in the desiccated skin niche is yet to be validated, the knowledge that lactic acid has a strong antimicrobial effect on clinical skin isolates of $S$. aureus can contribute to the development of lactic acid-containing products based on probiotic supernatants or lysates.

The proposed multifactorial L. rhamnosus GG mechanism of action and the role of different L. rhamnosus GG properties in our experimental set-up against $S$. aureus as a skin pathogen is summarised in Figure 6. Our data suggests that different molecules of L. rhamnosus GG can demonstrate anti-pathogenic action at different stages of $S$. aureus skin infection. For example, the presence of SpaCBA 
Lacticaseibacillus rhamnosus GG

properties
Probiotic adhesion and

immune receptor modulation

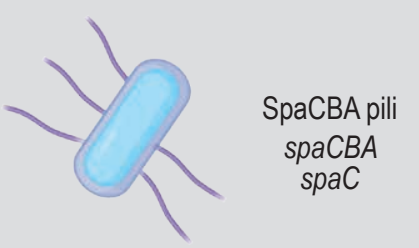

Cell surface molecules embedded in EPS $\triangle$ welE

Co-aggreration and autoaggregation spaCBA welE $\triangle m s p 1$
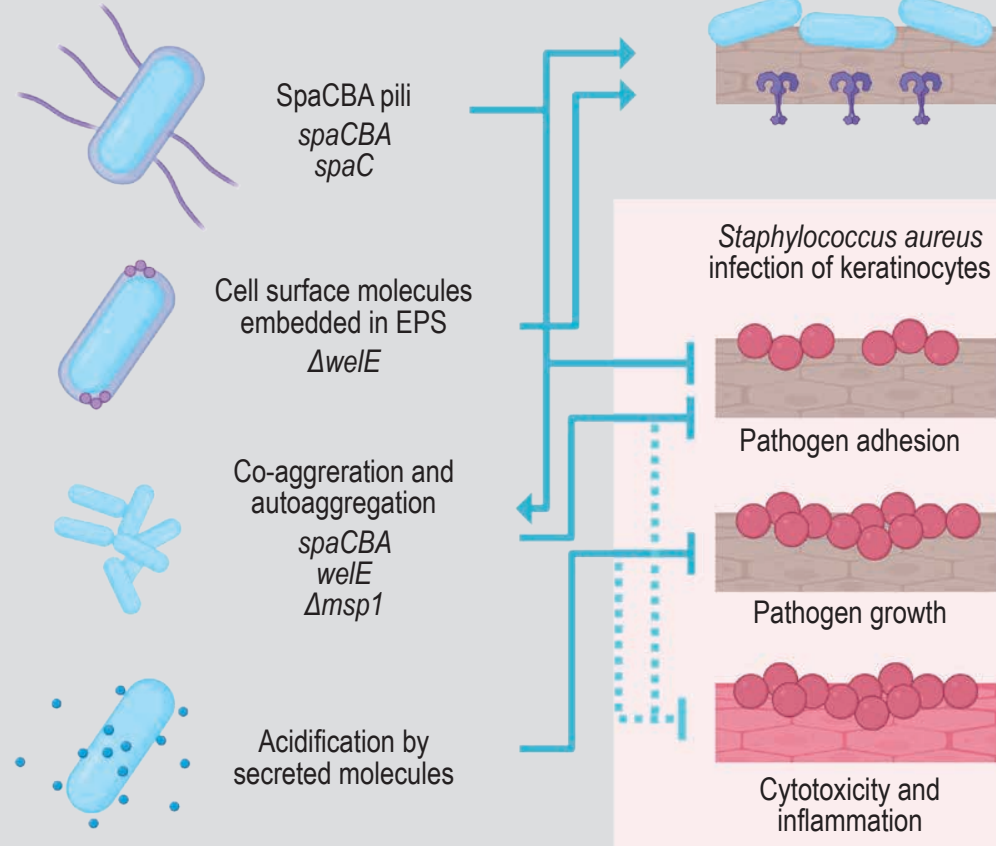

Staphylococcus aureus infection of keratinocytes

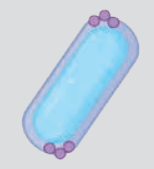

Figure 6. Proposed multifactorial Lacticaseibacillus rhamnosus GG mechanism of action against Staphylococcus aureus as a skin pathogen. Combined action of several molecular components produced by L. rhamnosus GG makes it an attractive candidate skin probiotic with potential action at different stages of $S$. aureus skin infection. Genotypes contributing to or enhancing the described properties are indicated in italics. Arrows indicate promotion, $T$ lines indicate inhibition and dotted lines indicate putative indirect effects.

pili ensures $L$. rhamnosus GG adhesion to keratinocytes and prevents initial adhesion of $S$. aureus (likely through host adhesion site blocking and partially co-aggregation), while secreted acidic components prevent further S. aureus growth. Ultimately, the combination of these and other probiotic mechanisms that are yet to be explored would result in less cytotoxicity, inflammation and cell death and in the host.

\section{Conclusions}

In conclusion, we showed that protection of human keratinocytes from S. aureus by live L. rhamnosus GG is multifactorial and involves both surface and secreted molecules. Adhesive probiotic surface molecules, such as the SpaCBA pili, play a key role during the initial colonisation stage, while soluble secreted factors become more important during long-term interactions of $S$. aureus with the host. The positive anti-inflammatory effects of $L$. rhamnosus on keratinocytes during long-term co-incubation with $S$. aureus are likely not purely immunostimulatory, and involve inhibition of both $S$. aureus growth and adhesion to host cells. The SpaCBA pili and cell wall EPS were identified as likely key factors in the probiotic-pathogenhost interactions, and involvement of other cell surface molecules embedded in EPS is hypothesised. Future in vivo studies will validate whether these mechanisms play an equally important role in the environment of the skin niche and against other clinical $S$. aureus isolates.

\section{Conflict of interest}

The authors declare no conflict of interest.

\section{Acknowledgements}

We would like to thank Abigail Elias and Cécile El-Chami for their technical support and insightful discussions. We would also like to express our gratitude to Mariya Petrova, Tine Verhoeven and Camille Allonsius for providing several Lacticaseibacillus rhamnosus GG mutants used in this study, and the guidance with their experimental implementation. We also thank Prof. Andrew McBain and Prof. Alexander Horswill for providing the S. aureus isolates. IS was partially supported by a personal research stay travel grant from FWO-Vlaanderen K224618N. 


\section{References}

Allonsius, C.N., Van den Broek, M.F.L., De Boeck, I., Kiekens, S., Oerlemans, E.F.M., Kiekens, F., Foubert, K., Vandenheuvel, D., Cos, P., Delputte, P. and Lebeer, S., 2017. Interplay between Lactobacillus rhamnosus GG and Candida and the involvement of exopolysaccharides. Microbial Biotechnology 10: 1753-1763. https://doi.org/10.1111/1751-7915.12799

Allonsius, C.N., Vandenheuvel, D., Oerlemans, E.F.M., Petrova, M.I., Donders, G.G.G., Cos, P., Delputte, P. and Lebeer, S., 2019. Inhibition of Candida albicans morphogenesis by chitinase from Lactobacillus rhamnosus GG. Scientific Reports 9: 2900. https://doi.org/10.1038/ s41598-019-39625-0

Burgain, J., Scher, J., Francius, G., Borges, F., Corgneau, M., RevolJunelles, A.M., Cailliez-Grimal, C. and Gaiani, C., 2014. Lactic acid bacteria in dairy food: Surface characterization and interactions with food matrix components. Advances in Colloid and Interface Science 213: 21-35. https://doi.org/10.1016/j.cis.2014.09.005

Burkholder, K.M. and Bhunia, A.K., 2009. Salmonella enterica serovar Typhimurium adhesion and cytotoxicity during epithelial cell stress is reduced by Lactobacillus rhamnosus GG. Gut Pathogens 1: 14 . https://doi.org/10.1186/1757-4749-1-14

Claes, I.J.J., Schoofs, G., Regulski, K., Courtin, P., Chapot-Chartier, M.-P., Rolain, T., Hols, P., Von Ossowski, I., Reunanen, J., De Vos, W.M., Palva, A., Vanderleyden, J., De Keersmaecker, S.C.J. and Lebeer, S., 2012. Genetic and biochemical characterization of the cell wall hydrolase activity of the major secreted protein of Lactobacillus rhamnosus GG. PLoS ONE 7: e31588-e31588. https:// doi.org/10.1371/journal.pone.0031588

Collado, M.C., Meriluoto, J. and Salminen, S., 2008. Adhesion and aggregation properties of probiotic and pathogen strains. European Food Research and Technology 226: 1065-1073. https://doi. org/10.1007/s00217-007-0632-x

De Keersmaecker, S.C., Verhoeven, T.L., Desair, J., Marchal, K., Vanderleyden, J. and Nagy, I., 2006. Strong antimicrobial activity of Lactobacillus rhamnosus GG against Salmonella typhimurium is due to accumulation of lactic acid. FEMS Microbiology Letters 259: 89-96. https://doi.org/10.1111/j.1574-6968.2006.00250.x

Gorbach, S.L., 1996. The discovery of Lactobacillus GG. Nutrition Today 31: 5S.

Kankainen, M., Paulin, L., Tynkkynen, S., Von Ossowski, I., Reunanen, J., Partanen, P., Satokari, R., Vesterlund, S., Hendrickx, A.P.A., Lebeer, S., De Keersmaecker, S.C.J., Vanderleyden, J., Hämäläinen, T., Laukkanen, S., Salovuori, N., Ritari, J., Alatalo, E., Korpela, R., Mattila-Sandholm, T., Lassig, A., Hatakka, K., Kinnunen, K.T., Karjalainen, H., Saxelin, M., Laakso, K., Surakka, A., Palva, A., Salusjärvi, T., Auvinen, P. and De Vos, W.M., 2009. Comparative genomic analysis of Lactobacillus rhamnosus GG reveals pili containing a human-mucus binding protein. Proceedings of the National Academy of Sciences of the USA 106: 17193-17198. https:// doi.org/10.1073/pnas.0908876106

Kiekens, S., Vandenheuvel, D., Broeckx, G., Claes, I., Allonsius, C., De Boeck, I., Thys, S., Timmermans, J.-P., Kiekens, F. and Lebeer, S., 2019. Impact of spray-drying on the pili of Lactobacillus rhamnosus GG. Microbial Biotechnology 12: 849-855. https://doi. org/10.1111/1751-7915
Kim, H.G., Gim, M.G., Kim, J.Y., Hwang, H.J., Ham, M.S., Lee, J.M., Hartung, T., Park, J.W., Han, S.H. and Chung, D.K., 2007. Lipoteichoic acid from Lactobacillus plantarum elicits both the production of interleukin-23p19 and suppression of pathogenmediated interleukin-10 in THP-1 cells. FEMS Immunology and Medical Microbiology 49: 205-214. https://doi.org/10.1111/j.1574695X.2006.00175.x

Lambers, H., Piessens, S., Bloem, A., Pronk, H. and Finkel, P., 2006. Natural skin surface $\mathrm{pH}$ is on average below 5 , which is beneficial for its resident flora. International Journal of Cosmetic Science 28: 359-370. https://doi.org/10.1111/j.1467-2494.2006.00344.x

Lebeer, S., Bron, P.A., Marco, M.L., Van Pijkeren, J.P., O'Connell Motherway, M., Hill, C., Pot, B., Roos, S. and Klaenhammer, T., 2018a. Identification of probiotic effector molecules: present state and future perspectives. Current Opinion in Biotechnology 49: 217-223. https://doi.org/10.1016/j.copbio.2017.10.007

Lebeer, S., Claes, I., Tytgat, H.L.P., Verhoeven, T.L.A., Marien, E., Von Ossowski, I., Reunanen, J., Palva, A., De Vos, W.M., De Keersmaecker, S.C.J. and Vanderleyden, J., 2012. Functional analysis of Lactobacillus rhamnosus GG pili in relation to adhesion and immunomodulatory interactions with intestinal epithelial cells. Applied and Environmental Microbiology 78: 185-193. https://doi. org/10.1128/AEM.06192-11

Lebeer, S., Claes, I.J., Verhoeven, T.L., Vanderleyden, J. and De Keersmaecker, S.C., 2011. Exopolysaccharides of Lactobacillus rhamnosus GG form a protective shield against innate immune factors in the intestine. Microbial Biotechnology 4: 368-374. https:// doi.org/10.1111/j.1751-7915.2010.00199.x

Lebeer, S., Oerlemans, E., Claes, I., Wuyts, S., Henkens, T., Spacova, I., Van den Broek, M., Tuyaerts, I., Wittouck, S., De Boeck, I., Allonsius, C.N., Kiekens, F. and Lambert, J., 2018b. Topical cream with live lactobacilli modulates the skin microbiome and reduce acne symptoms. bioRxiv 463307. https://doi.org/10.1101/463307

Lebeer, S., Verhoeven, T.L.A., Francius, G., Schoofs, G., Lambrichts, I., Dufrêne, Y., Vanderleyden, J. and De Keersmaecker, S.C.J., 2009. Identification of a gene cluster for the biosynthesis of a long, galactose-rich exopolysaccharide in Lactobacillus rhamnosus GG and functional analysis of the priming glycosyltransferase. Applied and Environmental Microbiology 75: 3554-3563. https:// doi.org/10.1128/AEM.02919-08

Ledder, R.G., Timperley, A.S., Friswell, M.K., Macfarlane, S. and McBain, A.J., 2008. Coaggregation between and among human intestinal and oral bacteria. FEMS Microbiology Ecology 66: 630636. https://doi.org/10.1111/j.1574-6941.2008.00525.x

Malone, C.L., Boles, B.R., Lauderdale, K.J., Thoendel, M., Kavanaugh, J.S. and Horswill, A.R., 2009. Fluorescent reporters for Staphylococcus aureus. Journal of Microbiological Methods 77: 251-260. https://doi.org/10.1016/j.mimet.2009.02.011

Mandlik, A., Swierczynski, A., Das, A. and Ton-That, H., 2007. Corynebacterium diphtheriae employs specific minor pilins to target human pharyngeal epithelial cells. Molecular Microbiology 64: 111-124. https://doi.org/10.1111/j.1365-2958.2007.05630.x 
Mohammedsaeed, W., McBain, A.J., Cruickshank, S.M. and O'Neill, C.A., 2014. Lactobacillus rhamnosus GG inhibits the toxic effects of Staphylococcus aureus on epidermal keratinocytes. Applied and Environmental Microbiology 80: 5773-5781. https://doi.org/10.1128/ AEM.00861-14

Peral, M.C., Huaman Martinez, M.A. and Valdez, J.C., 2009. Bacteriotherapy with Lactobacillus plantarum in burns. International Wound Journal 6: 73-81. https://doi.org/10.1111/ j.1742-481X.2008.00577.x

Perea Vélez, M., Petrova, M.I., Lebeer, S., Verhoeven, T.L., Claes, I., Lambrichts, I., Tynkkynen, S., Vanderleyden, J. and De Keersmaecker, S.C., 2010. Characterization of MabA, a modulator of Lactobacillus rhamnosus GG adhesion and biofilm formation. FEMS Immunology and Medical Microbiology 59: 386-98. https:// doi.org/10.1111/j.1574-695X.2010.00680.x

Petrova, M.I., Imholz, N.C.E., Verhoeven, T.L.A., Balzarini, J., Van Damme, E.J.M., Schols, D., Vanderleyden, J. and Lebeer, S., 2016. Lectin-like molecules of Lactobacillus rhamnosus GG inhibit pathogenic Escherichia coli and Salmonella biofilm formation. PLoS ONE 11: e0161337-e0161337. https://doi.org/10.1371/journal. pone. 0161337

Prince, T., McBain, A.J. and O'Neill, C.A., 2012. Lactobacillus reuteri protects epidermal keratinocytes from Staphylococcus aureus-induced cell death by competitive exclusion. Applied and Environmental Microbiology 78: 5119-5126. https://doi.org/10.1128/ AEM.00595-12

Ramos, A.N., Sesto Cabral, M.E., Arena, M.E., Arrighi, C.F., Arroyo Aguilar, A.A. and Valdéz, J.C., 2015. Compounds from Lactobacillus plantarum culture supernatants with potential pro-healing and anti-pathogenic properties in skin chronic wounds. Pharmaceutical Biology 53: 350-358. https://doi.org/10.3109/13880209.2014.920037

Reid, G., Charbonneau, D., Erb, J., Kochanowski, B., Beuerman, D., Poehner, R. and Bruce, A.W., 2003. Oral use of Lactobacillus rhamnosus GR-1 and L. fermentum RC-14 significantly alters vaginal flora: randomized, placebo-controlled trial in 64 healthy women. FEMS Immunology and Medical Microbiology 35: 131-134. https:// doi.org/10.1016/S0928-8244(02)00465-0

Reid, G., Tieszer, C. and Lam, D., 1995. Influence of lactobacilli on the adhesion of Staphylococcus aureus and Candida albicans to fibers and epithelial cells. Journal of Industrial Microbiology 15: 248-253. https://doi.org/10.1007/BF01569832

Segers, M.E. and Lebeer, S., 2014. Towards a better understanding of Lactobacillus rhamnosus GG - host interactions. Microbial Cell Factories 13, Suppl. 1: S7. https://doi.org/10.1186/1475-2859-13S1-S7
Spacova, I., Lievens, E., Verhoeven, T., Steenackers, H., Vanderleyden, J., Lebeer, S. and Petrova, M.I., 2018. Expression of fluorescent proteins in Lactobacillus rhamnosus to study host-microbe and microbe-microbe interactions. Microbial Biotechnology 11: 317331. https://doi.org/10.1111/1751-7915.12872

Strober, W., 1997. Trypan blue exclusion test of cell viability. Current Protocols in Immunology 21: A.3B.1-A.3B.2. https://doi. org/10.1002/0471142735.ima03bs21

Tang, S.C. and Yang, J.H., 2018. Dual effects of alpha-hydroxy acids on the skin. Molecules 23: 863. https://doi.org/10.3390/ molecules23040863

Tytgat, H., 2015. Glycoproteins in probiotic bacteria - exploring the glycosylation potential of Lactobacillus rhamnosus GG by genomics and glycoproteomics. Doctoral dissertation, KU Leuven, Leuven, Belgium.

Van den Broek, M.F.L., De Boeck, I., Claes, I.J.J., Nizet, V. and Lebeer, S., 2018. Multifactorial inhibition of lactobacilli against the respiratory tract pathogen Moraxella catarrhalis. Beneficial Microbes 9: 429439. https://doi.org/10.3920/BM2017.0101

Vargas García, C.E., Petrova, M., Claes, I.J., De Boeck, I., Verhoeven, T.L., Dilissen, E., Von Ossowski, I., Palva, A., Bullens, D.M., Vanderleyden, J. and Lebeer, S., 2015. Piliation of Lactobacillus rhamnosus GG promotes adhesion, phagocytosis, and cytokine modulation in macrophages. Applied and Environmental Microbiology 81: 2050-2062. https://doi.org/10.1128/AEM.03949-14 Vesterlund, S., Karp, M., Salminen, S. and Ouwehand, A.C., 2006. Staphylococcus aureus adheres to human intestinal mucus but can be displaced by certain lactic acid bacteria. Microbiology 152: 1819-1826. https://doi.org/10.1099/mic.0.28522-0

Von Ossowski, I., Reunanen, J., Satokari, R., Vesterlund, S., Kankainen, M., Huhtinen, H., Tynkkynen, S., Salminen, S., De Vos, W.M. and Palva, A., 2010. Mucosal adhesion properties of the probiotic Lactobacillus rhamnosus GG SpaCBA and SpaFED pilin subunits. Applied and Environmental Microbiology 76: 2049-2057. https:// doi.org/10.1128/AEM.01958-09

Zárate, G. and Nader-Macias, M.E., 2006. Viability and biological properties of probiotic vaginal lactobacilli after lyophilization and refrigerated storage into gelatin capsules. Process Biochemistry 41: 1779-1785. https://doi.org/10.1016/j.procbio.2006.03.024

Zhang, Z., Zhou, Z., Li, Y., Zhou, L., Ding, Q. and Xu, L., 2016. Isolated exopolysaccharides from Lactobacillus rhamnosus GG alleviated adipogenesis mediated by TLR2 in mice. Scientific Reports 6: 36083. https://doi.org/10.1038/srep36083 
